\title{
Intermediate-term results of a nonresectional dynamic repair technique in 662 patients with mitral valve prolapse and mitral regurgitation
}

\author{
Gerald M. Lawrie, MD, Elizabeth A. Earle, JD, and Nan Earle, MS
}

\begin{abstract}
Objective: A nonresectional technique has been developed for repair of mitral leaflet prolapse causing mitral regurgitation. Polytetrafluoroethylene chordae are used for correction of edge misalignment of the prolapsed mitral leaflet. New chordal length is adjusted during progressive left ventricular inflation to systolic pressure. Annular sizing is determined dynamically after leaflet edge alignment is accomplished to produce an optimal zone of predefined leaflet apposition. The aim of this study was to document the 8- to 10-year durability of this nonresectional approach.
\end{abstract}

\begin{abstract}
Methods: From 1983 through 2008, 1121 consecutive patients had mitral valve repair on one service. Of these, 662 had repair of mitral leaflet prolapse. From 1983 until 1998, standard quadratic leaflet resection/plication was used in $72(11.1 \%)$ patients, similar but smaller resection in $93(14.1 \%)$ patients, and then smaller resection and polytetrafluoroethylene chordae in $24(3.7 \%)$ patients. All received Puig-Massana fully flexible rings (Shiley, Inc, Irvine, Calif). After 1998, no leaflet resections or valve replacements have been performed regardless of leaflet size in 566 consecutive patients. Of the 662 patients, the mean age was $62.6 \pm 14.1$ years, and $424(64.1 \%)$ patients were male. Coronary artery disease was present in $147(22.2 \%)$ patients and $33(5.0 \%)$ had prior coronary artery bypass. Leaflets corrected were as follows: anterior, 152 (23.0\%) patients; posterior, 427 (64.5\%); and both, $83(12.5 \%)$ Common pathologic characteristics of prolapsing valves were as follows: myxomatous, $332(50.2 \%)$ patients, degenerative, $83(12.5 \%)$, ischemic, $31(4.7 \%)$, and rheumatic, $29(4.4 \%)$.

Results: Perioperative mortality was $2.9 \%$ (19/662) overall and $0.49 \%(2 / 414)$ for isolated repair. Freedom from reoperation at 10 years (Kaplan-Meier) was $90.1 \%$ and freedom from significant mitral regurgitation (echocardiography) was $93.9 \%$.

Conclusions: This study confirms that mitral regurgitation from mitral leaflet prolapse can be repaired in all cases by a nonresectional technique provided that accurate dynamic evaluation of chordal length and annular sizing is achieved. The intermediate-term results are durable. (J Thorac Cardiovasc Surg 2011;141:368-76)
\end{abstract}

After the first open repair of a mitral valve for mitral regurgitation (MR) by McGoon, ${ }^{1}$ a variety of surgical approaches were developed. They were directed primarily at correcting abnormalities of the leaflets, chordae tendineae, and mitral annulus. The pioneering work of Carpentier ${ }^{2,3}$ led to the first systematic and reproducible techniques based on detailed anatomic and pathologic studies. Recent reports have documented some limitations of these techniques, including difficulty in repairing all types of myxomatous disease and significant rates of recurrent MR on echocardiography at 10 years of follow-up. ${ }^{4-7}$

Over the past 40 years, a substantial body of experimental data from the dog and other animal models has accumulated. ${ }^{8,9}$ Progressive refinements of clinical echocardiography have

From the Heart Valve Institute, Methodist DeBakey Heart and Vascular Center, Houston, Tex.

Disclosures: Authors have nothing to disclose with regard to commercial support.

Received for publication Sept 30, 2009; revisions received Feb 3, 2010; accepted for publication Feb 17, 2010; available ahead of print April 23, 2010.

Address for reprints: Gerald M. Lawrie, MD, 6560 Fannin, Suite 1842, Houston, TX 77030 (E-mail: gmlawrie@att.net).

$0022-5223 / \$ 36.00$

Copyright (C) 2011 by The American Association for Thoracic Surgery doi:10.1016/j.jtcvs.2010.02.044 led to better understanding of the remarkably complex and dynamic function of the mitral valve in the beating heart.

On the basis of these newer insights, we ${ }^{10}$ developed a nonresectional technique of mitral valve repair in which accurate dynamic adjustment of polytetrafluoroethylene (PTFE) chordal length and annular dimensions is emphasized. This approach uses intraoperative dynamic simulation of phases of the cardiac cycle by progressive left ventricular distention with pressurized saline to allow maximum restoration and use of the normal dynamic mitral valve functions that promote systolic leaflet apposition and reduce leaflet and chordal stress. We hypothesized that optimal adjustment of mitral annular dimensions and mitral leaflet edge position during aortic root distention would also avoid systolic anterior motion of the mitral leaflet (SAM). In this prospective study, we report on our experience in a series of patients with MR from mitral valve prolapse disease in whom $100 \%$ reparability was demonstrated in a large series of patients with a low incidence of late recurrent MR. This subgroup was chosen inasmuch as it represents a commonly reported and easily identifiable category for purposes of comparison with other techniques. 


\section{Abbreviations and Acronyms \\ $\mathrm{MR}=$ mitral regurgitation \\ PTFE $=$ polytetrafluoroethylene \\ $\mathrm{SAM}=$ systolic anterior motion of the mitral leaflet}

\section{PATIENTS AND METHODS}

This prospective study was approved by our institutional review board and was compliant with the Health Insurance Portability and Accountability Act. The patients in this study all had a diagnosis of MR from mitral valve leaflet prolapse. The diagnosis was confirmed by preoperative transthoracic and/or transesophageal echocardiography and intraoperatively by transesophageal echocardiography and direct inspection during surgery. Between 1983 and 2008, a total of 1368 patients underwent mitral valve prosthetic replacement or repair for MR. Of these patients, 1121 underwent mitral valve repair between 1983 and 2008. From this group, 662 patients were identified who had mitral valve leaflet prolapse as the cause of their MR. The preoperative clinical, angiographic, and echocardiographic features of these 662 patients are shown in Table 1. The most common pathologic condition treated was myxomatous disease. The other major pathologic group we have termed "calcific degeneration of the mitral valve." This group is older and has a higher prevalence of coronary artery disease and diabetes, previous myocardial infarctions, and lower ejection fractions. Their leaflets have normal or mildly reduced dimensions but often are somewhat fibrotic and have lipid and calcific infiltration, especially in the posterior annulus. They typically have isolated chordal rupture. Their annular dimensions are usually in the mid 30-mm range. The patients with ischemic disease all had localized leaflet prolapse secondary to papillary muscle infarction with either rupture of the tip or elongation of the entire muscle, usually posteromedial. The A2,3 segments were most commonly affected. These patients were a separate group from the patients with normal leaflets, chordae, and papillary muscles but with the more commonly seen "restrictive" MR from inferior wall myocardial infarction. The technique reported here was developed in the first 165 patients undergoing repair for mitral valve leaflet prolapse between 1983 and 1998. In the first $72(11.1 \%)$ patients, classic quadratic resection as described by Carpentier $^{3}$ and David and associates ${ }^{4}$ was used. ${ }^{3,4}$ In the next $93(14.1 \%)$ patients, smaller quadratic resections were used. Subsequently, PTFE chordae were used, initially to supplement smaller leaflet resections in $24(3.7 \%)$ patients and then as the sole technique. By 1995 this became our preferred technique. Chordal length was adjusted in the arrested empty heart by matching leaflet edge position to adjacent and opposite "normal" leaflets. In the earlier experience, annuloplasty sizing was also performed in the empty heart by measurement of the circumference of the annulus. A fully flexible ring (Puig-Massana fully flexible rings; Shiley, Inc, Irvine, Calif) attached to the annulus with a running suture has been used throughout this experience. The repair was tested after completion with saline insufflation as recommended by Carpentier ${ }^{3}$ and David and associates. ${ }^{4}$ After the observation of reproducible annular and leaflet motion changes with progressive cardiac inflation, we determined that the use of inflation could be extended to assist in the precise sizing of chordae and the annuloplasty. In this way, the relationship of the leaflets and their zone of apposition, as well as the movement of the leaflets into the left ventricular cavity and the expansion of the aortic root, could be simulated. After 1998, the surgical technique described below was used in its entirety in all patients. No leaflet resections have been performed, regardless of leaflet size. All patients underwent echocardiography before hospital discharge. The severity of MR was graded as none, mild, moderate, moderately severe, or severe. Patient follow-up was by annual patient questionnaires, telephone calls, reports from cardiologists, and examination of the Social Security Deaths Index at Rootsweb. ancestry.com.

\section{Statistical Analysis}

The data were recorded prospectively on custom forms and entered into a custom database. The database, which was designed in FileMaker (FileMaker Pro v 7.0v2), resides on a Pentium III Personal Computer running Windows 2000 Profession, v5.0.2195 with Service Pack 4 (Microsoft Corp, Redmond, Wash). Data analyses were carried out in S-Plus v 6.2, Professional Edition (Insightful Corporation, Seattle, Wash). The variables of interest were imported into S-Plus via an intermediate Excel file (Microsoft Excel 2003 [11.6560.8036SP2]). Formal statistical analyses carried out in S-Plus were as follows: $\chi^{2}$ analyses for categorical data, analysis of variance for continuous variables, and Cox proportional hazards analysis for predictors of survival and freedom from reoperation, as well as Kaplan-Meier survival analyses and their resulting graphs. There was no adjustment for multiple tests.

\section{Surgical Technique of Nonresectional Dynamic Repair}

The chest is entered through a full median sternotomy, a limited lower sternal incision, or more recently with the da Vinci robotic assisted approach (da Vinci Surgical System; Intuitive Surgical, Inc, Mountain View, Calif) via the right lateral chest wall through a $20-\mathrm{mm}$ working port. The left atrium is entered through an interatrial incision and the mitral valve is exposed. Stay stitches of 2-0 polypropylene are placed at the following sites: 2 stays placed anterior to the commissures into the right and left fibrous trigones and 2 or 3 into the posterior mitral annulus. Artificial chordal replacement with No. 5 PTFE sutures is begun by placing the PTFE sutures through the lower third of the anterolateral and posteromedial papillary muscles in a figure-of- 8 fashion (Figure 1, $A$ ). The PTFE chordae are brought through the free edge of the prolapsing segment as a rolling suture (Figure 1, $B$ ). For the commonly encountered prolapsing P2 segment with a basal annular attachment dimension of 2 to $2.5 \mathrm{~cm}, 4$ chordae ( 2 pairs) are used: 2 from the anterolateral and 2 from the posteromedial papillary muscles. When larger leaflets are encountered, a third pair of chordae are placed from a separate site on either the anterolateral or posteromedial papillary muscles. Experience suggests that the leaflet edges should be supported by PTFE chordae every 8 to $10 \mathrm{~mm}$. Dots are placed with a marker pen along the upper margins of the rough zones to mark the desired line of leaflet apposition. If this transition is not identifiable, then the dots are placed 5 to $10 \mathrm{~mm}$ from the free edges of the leaflets, depending on the degree of leaflet enlargement. The left ventricle is inflated with saline at $4 \mathrm{~L} / \mathrm{min}$ from a mechanized laparoscopic suction irrigator to a pressure of 80 to $90 \mathrm{~mm} \mathrm{Hg}$. During this maneuver, the ascending aorta is allowed to vent passively through a Bengash aspirating needle to avoid air embolism of the right coronary artery. The ascending aorta is checked to ensure that it is distended. The lengths of the PTFE chordae are adjusted to align the edges of the leaflets using the relative height of the lines of dots on each leaflet as a guide. The posterior annulus is displaced forward toward the aortic root using the previously placed posterior annular stitches while the ventricle is inflated. This simulates the effects of the end-diastolic left atrial contraction of $25 \%$ reduction of the posterior annulus. The rough zones as defined by the dots and aligned by the adjusted chordae come together toward each other. The leaflets descend into the cavity of the left ventricle. The lines of dots on both leaflets indicate progressive increase in leaflet apposition with continued inflation. The mitral annulus descends away from the surgeon toward the apex. "Mitral locking" is present at this point with the leaflets forcibly apposed to each other (Figure 1,C). Leaflet alignment and apposition are checked with another inflation with saline, and the PTFE chordae are further adjusted as needed. At this point, the mitral orifice is sized according to the circumference of the mitral annulus in this position with standard annuloplasty ring sizers (Figure 1,C). A fully flexible adjustable ring is chosen of this size. This will maintain permanently the zone of leaflet apposition established by these maneuvers.

The PTFE is tied down and the knot is locked with a 6-0 polypropylene stitch tied over the end of the PTFE knot to prevent sliding of the PTFE knots. The annuloplasty ring is secured to the annulus with the previously 
TABLE 1. Preoperative variables in 662 patients with MR from mitral valve prolapse

\begin{tabular}{lc}
\hline Age, y (mean \pm SD) & $62.6 \pm 14.1$ \\
Sex, percent male & $64.1 \%(424 / 662)$ \\
Diabetes & $14.1 \%(72 / 511)$ \\
Prior myocardial infarction & $9.8 \%(65 / 662)$ \\
Coronary artery disease & $22.2 \%(147 / 662)$ \\
Prior coronary artery bypass & $5.0 \%(33 / 662)$ \\
Preop atrial fibrillation & $30.8 \%(204 / 662)$ \\
NYHA class III or IV & $55.9 \%(248 / 444)$ \\
Preop ejection fraction $(\%)$ & $59.5 \pm 11.2$ \\
Preop MR, grade 3 or 4 & $92.0 \%(609 / 662)$ \\
Preop annular dimensions $(\mathrm{mm})$ & $49.6 \pm 6.1$ \\
Anterior leaflet prolapse & $23.0 \%(152 / 662)$ \\
Posterior leaflet prolapse & $64.5 \%(427 / 662)$ \\
Bileaflet prolapse & $12.5 \%(83 / 662)$ \\
Pathology & \\
Myxomatous & $50.2 \%(332 / 662)$ \\
Calcific degenerative & $12.5 \%(83 / 662)$ \\
Both degenerative and & $21.6 \%(143 / 662)$ \\
$\quad$ myxomatous & \\
Ischemic & $4.7 \%(31 / 662)$ \\
Rheumatic & $4.4 \%(29 / 662)$ \\
Endocarditis & $3.5 \%(23 / 662)$ \\
Other & $3.2 \%(21 / 662)$ \\
\hline
\end{tabular}

NYHA, New York Heart Association; $M R$, mitral regurgitation.

placed trigonal 2-0 sutures in continuous fashion. The valve is again tested by saline insufflation of the left ventricle. The marking dots and PTFE knots now should be observed to be buried in the line of apposition (Figure 1,D). The left atrium is closed. The air is evacuated, warm blood cardioplegic solution given, and the aorta is unclamped. The operation is completed and the repair is checked by transesophageal echocardiography. Further technical details, including videos of this technique by open and robotic exposures are available at www.geraldlawriemd.com.

\section{RESULTS}

The repair rate was defined as the number of repairs performed divided by the total number of mitral repairs and replacements and expressed as a percentage. From 1983 until 1998 , the 165 patients who had successful repairs represented $66.5 \%$ of the group of all patients who were referred for mitral valve surgery for MR. After 1998 the overall repair rate for all causes was $88.5 \%$. For the 497 patients with MR from mitral valve leaflet prolapse operated on since 1998 , it has been $100 \%$. Other associated procedures performed were coronary artery bypass, $119(18.0 \%)$; aortic valve replacement $19(2.9 \%)$, and tricuspid valve replacement, 25 (3.8\%) (Table 2). Perioperative mortality overall was $17(2.6 \%)$ of 662 patients, and for isolated repair, 2 $(0.49 \%)$ of 404 patients. For isolated posterior leaflet repair, perioperative mortality was $1(0.37 \%)$ of 270 patients.

All patients underwent postoperative echocardiography before discharge. For the overall group of 662 patients, preoperatively, $599(90.5 \%)$ patients had severe or moderately severe MR, $62(9.4 \%)$ had moderate, and $1(0.2 \%)$ had mild MR (a patient with endocarditis). After surgery but before discharge, $1(0.2 \%)$ patient had severe MR, $5(0.8 \%)$ had moderately severe, $23(3.5 \%)$ had moderate, $139(21 \%)$ had mild, and 504 (76.1\%) had no MR. In follow-up, of $469(71 \%)$ patients with late echocardiograms, $12(2.6 \%)$
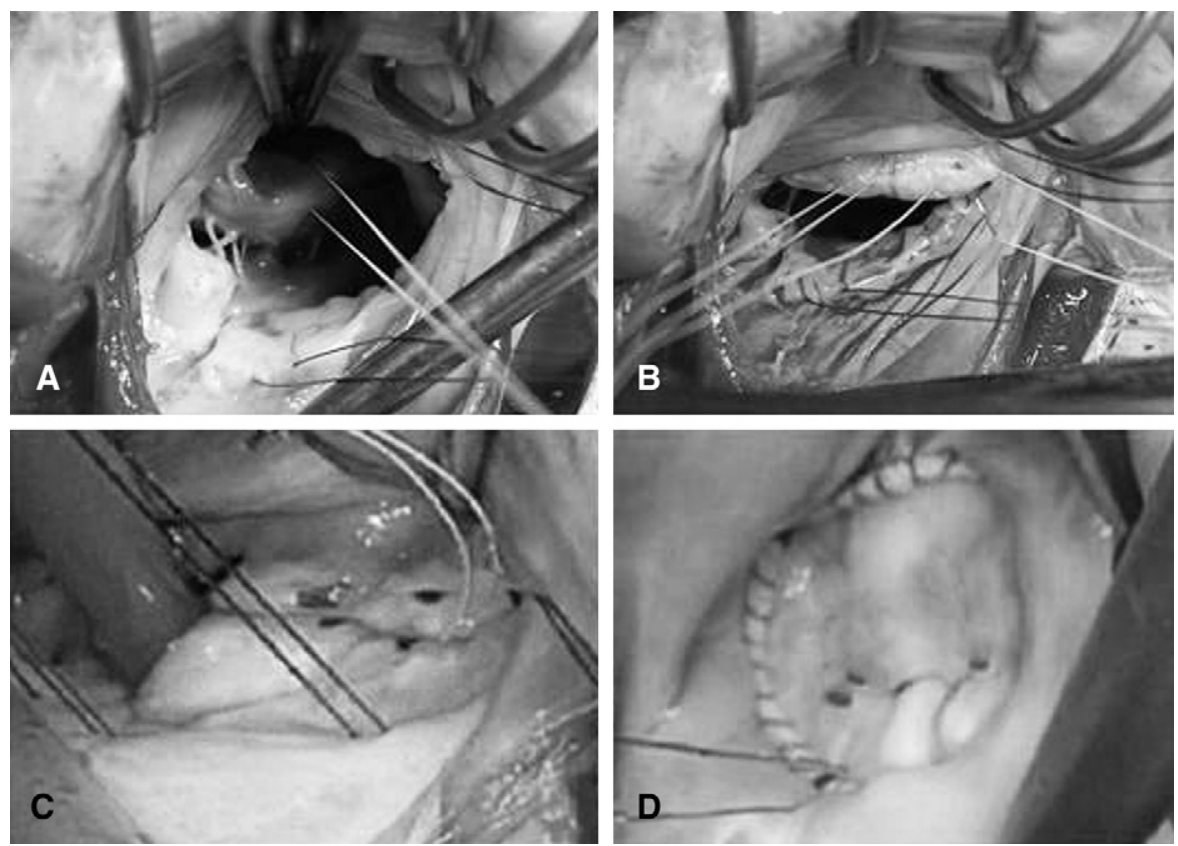

FIGURE 1. Surgical technique of the "American Correction." A, Polytetraflluoroethylene chordae are placed in the base of the papillary muscle. B, Chordae are placed in the free edge of the leaflet. C, The ventricle is inflated. The posterior annulus is pulled forward and the zone of apposition forms. The annuloplasty ring is chosen to match the annular circumference at this point. D, The ring is secured with a continuous suture of 2-0 polypropylene. 
TABLE 2. Surgical procedures and outcome in 662 patients

\begin{tabular}{lc}
\hline Annuloplasty ring size used (mm) & $28.91 \pm 2.82$ \\
Preoperative annular area (3D) & $18.5 \pm 4.6 \mathrm{~cm}^{2}$ \\
Postoperative annular area (3D) & $6.6 \pm 1.7 \mathrm{~cm}^{2}$ \\
No. chordae replaced/patient & $4.3 \pm 1.65(\mathrm{range}, 2-12)$ \\
No. anterior chordae replaced & $3.62 \pm 1.52$ \\
No. posterior chordae replaced & $4.0 \pm 1.39$ \\
Concurrent CAB & $18.0 \%(119 / 662)$ \\
Concurrent AVR & $2.9 \%(19 / 662)$ \\
Concurrent TVR & $3.8(25 / 662)$ \\
Pump time, min (median, range) & \\
Isolated mitral valve repair & $70(50-187)$ \\
Combined & $101(72-174)$ \\
Clamp time, min (median,range) & \\
Isolated mitral valve repair & $58(38-123)$ \\
Combined & $86(60-131)$ \\
Perioperative mortality in 662 patients & \\
Overall & $2.9 \%(19 / 662)$ \\
Isolated mitral repair & $0.49 \%(2 / 404)$ \\
Isolated posterior leaflet repair & $0.37 \%(1 / 270)$ \\
Hospital stay, days & $11.32 \pm 10.56$ \\
Predischarge MR, grade 3 or 4 & $1.4 \%(6 / 417)$ \\
Follow-up, mo & $51.37 \pm 50.20$ \\
\hline $3 D$, Three-dimensional; $C A B$, coronary artery bypass; $A V R$, aortic valve replacement; \\
$T V R$, tricuspid valve replacement; $M R$, mitral regurgitation.
\end{tabular}

patients had severe MR, $11(2.3 \%)$ had moderately severe, $46(9.8 \%)$ had moderate, $150(32.0 \%)$ had mild, and 250 $(53.3 \%)$ had no MR. The mean late follow-up interval was $1.8 \pm 3.0(\mathrm{SD})$ years for the 662 patients. There was more than 5 years of follow-up in 361 patients and more than 10 years in 120 patients. The time-related freedom from moderately severe or severe MR on echocardiography analyzed by the Kaplan-Meier technique is shown in Figure 2 (right panel). For the subgroup of 391 patients with myxomatous or calcific degenerative disease only, the mean follow-up interval was $3.4 \pm 3.8$ (SD) years. Preoperatively, $352(76.5 \%)$ of these patients had severe MR, 82 $(17.82 \%)$ had moderately severe, $19(4.1 \%)$ moderate, and $1(0.2 \%)$ mild. Before discharge, $1(0.25 \%)$ patient had severe MR, $3(0.65 \%)$ had moderately severe, 19 $(4.13 \%)$ had moderate, $108(23 \%)$ had mild, and 329 $(72 \%)$ had no MR. In follow-up, $9(2.3 \%)$ patients had severe MR, 7 (1.8\%) had moderately severe, $37(9.46 \%)$ had moderate, $114(29.16 \%)$ had mild, and $224(57.29 \%)$ had no MR. These results are summarized in Figure 3.

Freedom from reoperation at 10 years was $90.1 \%$ (Kaplan-Meier) (Figure 2, left). Durability was similar for anterior, posterior, and bileaflet repair $(P=$ not significant). Of the 165 patients operated on before 1998, $13(7.9 \%)$ required reoperation at a mean interval of $4.2 \pm 3.9$ (SD) years. Among the 13 with reoperations performed before 1998, 1 patient had a ruptured papillary muscle 3 years postoperatively; 5 patients with rheumatic valve disease required reoperation for late development of mitral stenosis; 2 patients who had repair without annuloplasty rings required reoperation for annular dilatation and progressive MR; 1 patient initially operated on for endocarditis required early reoperation with a prosthetic valve; 1 patient had reoperation after 5 years for failed triangular resection of the anterior leaflet.

A total of 497 patients were operated on for mitral valve leaflet prolapse from 1998 through 2008, after leaflet resection had been discontinued and dynamic repair became completely standardized. Reoperation was required in $17(3.4 \%)$ $(P<.05)$ of these 497 patients after the initial repair at a mean interval of $2.3 \pm 1.9(\mathrm{SD})$ years. Of these, 4 required reoperation for hemolysis from small, high-velocity jets.
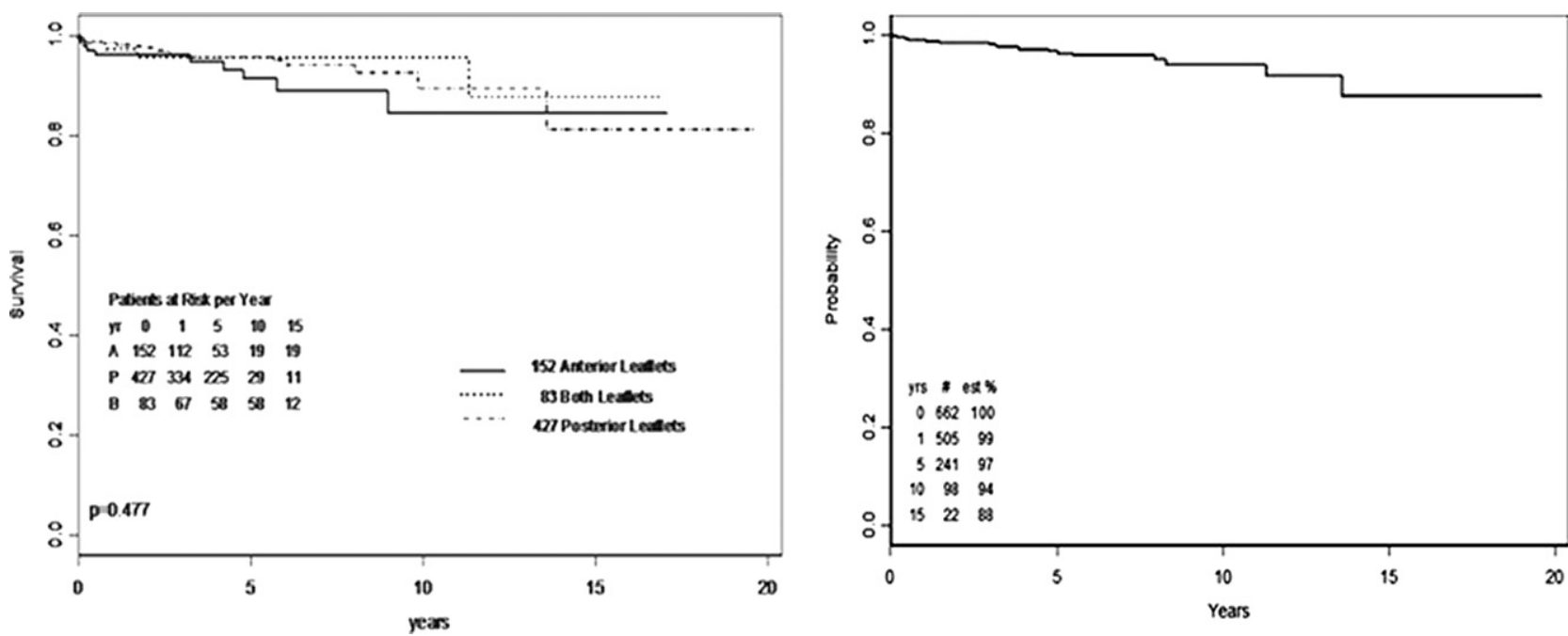

FIGURE 2. Left, Results of Kaplan-Meier analysis of freedom from reoperation up to 10 years after the initial operation, according to the leaflet repaired. Right, Results of Kaplan-Meier analysis of freedom from moderately severe or severe mitral regurgitation 10 years after the mitral operation. The number of patients at risk for each interval is shown in the tables on the figures. 
Mitral Regurgitation in Mitral Valve Repair Patients with Myxomatous or Degenerative Pathdogy

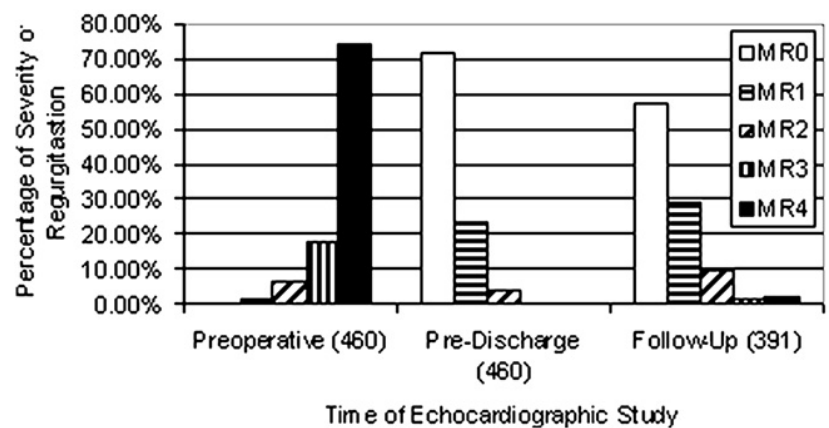

FIGURE 3. Comparison of severity of mitral regurgitation assessed by echocardiography preoperatively, before discharge, and at late follow-up.

Three underwent successful repeat repair and 1 received a mechanical prosthesis in another center. Two patients were borderline candidates for the initial repair. One had extensive annular calcification involving the leaflets and had a prosthetic replacement 12 days postoperatively. The other had advanced rheumatic changes and required prosthetic replacement after 3 months. One patient with a good result for 9 years required reoperation on an emergency basis with a flail posterior leaflet from a ruptured papillary muscle after acute myocardial infarction. A prosthetic replacement was required. One patient required prosthetic replacement for endocarditis 9 months after an initially successful repair. The remaining 6 patients all had received partial annuloplasty rings at the original operation and had recurrent annular dilatation. All had a successful second repair with a smaller, full ring annuloplasty and chordal revision.

Thus, the rate of reoperation has been lower $(3.4 \%$ vs $7.9 \% ; P<.05)$ and the need for prosthetic replacement at the time of reoperation has been lower after leaflet resection was discontinued. Of the 13 procedures before 1998, $7(53.8 \%)$ were prosthetic replacements, and after 1998, 5 $(29.3 \%)$ of 17 were prosthetic replacements $(P<.05)$.

No patient has required reoperation for SAM since before 1998. Mild chordal SAM without MR has been observed transiently intraoperatively in approximately $20 \%$ of patients but has never caused a clinical problem. In 5 patients, all with hypertrophic cardiomyopathy and small left ventricular cavities, SAM with mild-to-moderate MR persisted perioperatively but ultimately responded to medical therapy before discharge. A subgroup of 60 consecutive patients from the 662 patients was enrolled in a prospective study using 3-dimensional echocardiography perioperatively and for 6 months' follow-up evaluation. At 6 months, 6 patients had chordal SAM. In 4 patients there was no MR, in 1 trace MR, and in 1 mild MR (unpublished data).

The results of Cox analysis for factors predictive of mortality and factors predictive of need for reoperation are shown in Table 3.

\section{DISCUSSION}

Myxomatous degeneration of the mitral valve is the most common cause of severe MR. Once thought to be unsuitable for mitral valve repair, it is now known to be the condition with the highest success rates after repair. ${ }^{11}$

The natural history of this condition has been widely described. ${ }^{12-16}$ There are striking pathoanatomic changes causing enlargment of the leaflets and chordal elongation, but the mitral annular dimensions and function usually are preserved and the large majority of patients do not have MR. Despite the large leaflets, elongated chordae, and normal annular dimensions, SAM is not seen in these patients. MR from myxomatous valves is almost always accompanied by mitral annular dilatation. Severe MR is not seen in patients with intact chordae without mitral annular dilatation. ${ }^{13-17}$ In population-based studies, leaflet dimensions in patients without MR are similar to those seen in patients with more severe MR. ${ }^{15,17}$ Annular dilatation produces multiple deleterious effects on mitral valve function. These include decreased or absent leaflet apposition, decrease in the late diastolic annular area reduction from $25 \%$ to $15 \%$, marked increase in leaflet stress, marked increase in chordal stress, and systolic and diastolic flattening of the saddle shape of the annulus. ${ }^{18-20}$

The dynamic behavior of the mitral annulus during the cardiac cycle was reported first by Tsakiris and associates ${ }^{8}$

TABLE 3. Results of Cox regression A

\begin{tabular}{|c|c|c|c|c|}
\hline & Relative risk & Lower $95 \%$ CI & Upper $95 \%$ CI & $P$ value \\
\hline \multicolumn{5}{|l|}{ Relative risk of mortality } \\
\hline Age & 1.066 & 1.043 & 1.090 & $<.0001$ \\
\hline Both leaflets repaired & 0.372 & 0.142 & 0.973 & .0440 \\
\hline Degenerative pathology & 3.296 & 1.510 & 7.196 & .0027 \\
\hline Myxomatous pathology & 2.097 & 1.108 & 3.969 & .0023 \\
\hline Neither myxomatous nor degenerative pathology & 3.533 & 1.722 & 7.250 & .0006 \\
\hline Isolated repair & 0.501 & 0.260 & 0.965 & .0390 \\
\hline \multicolumn{5}{|l|}{ Relative risk of reoperation at 10 years } \\
\hline Neither myxomatous nor degenerative pathology & 8.73 & 1.739 & 43.83 & .0085 \\
\hline Preoperative EF & 1.06 & 0.998 & 1.13 & .0570 \\
\hline
\end{tabular}

$C I$, Confidence interval; $E F$, ejection fraction. 


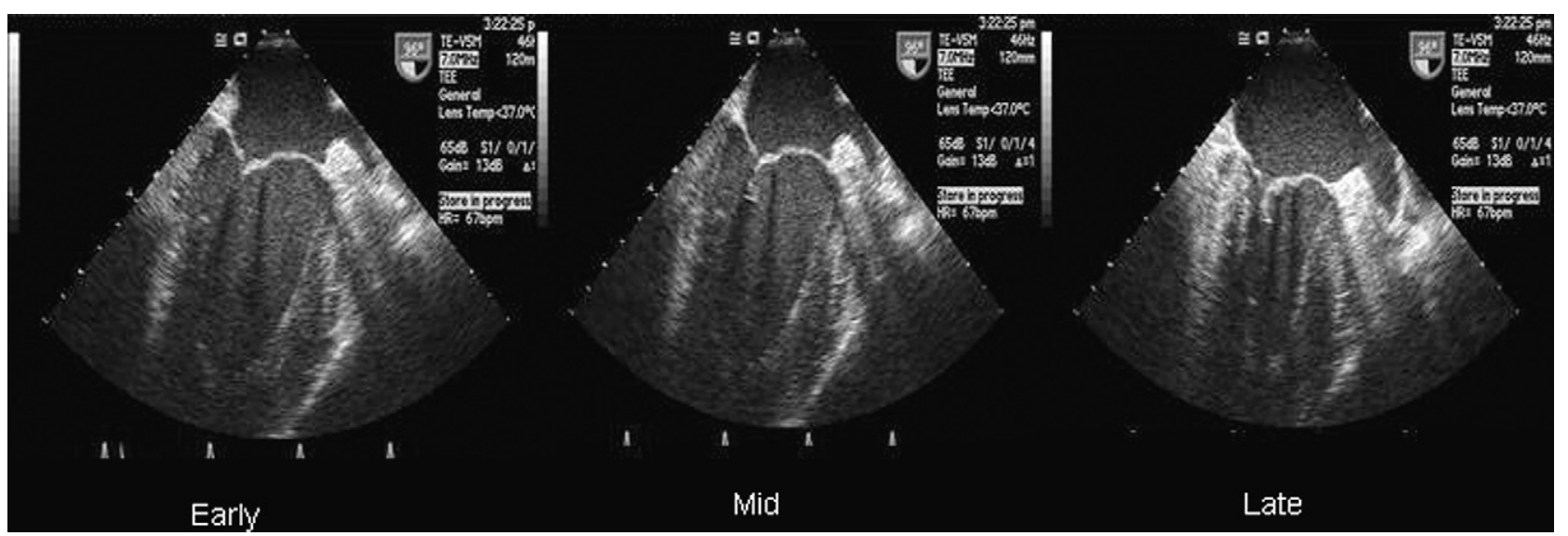

FIGURE 4. Systolic still frames from a study of a normal patient by transesophageal echocardiography. As the annular and left ventricular dimensions diminish progressively during systole, there is notable increase in the extent of mitral leaflet apposition. The annulus also moves toward the apex.

in the dog model. Subsequent studies in animals and humans have confirmed and expanded these findings. ${ }^{9}$ The normal mitral annulus rises upward toward the left atrium during diastole and downward toward the left ventricular apex during systole. It travels about 1.5 to $2 \mathrm{~cm}$. During atrial contraction at the end of diastole, the mitral annular area is reduced by about $25 \%$, and further reduction of $5 \%$ to $10 \%$ occurs during systole. This area reduction is achieved by a combination of mechanisms. There is sphincteric contraction of left atrial and ventricular circular muscle fibers. In addition, there are complex changes in the 3-dimensional disposition of the annulus owing to inward folding along the intercommisural and the septolateral axes. These changes are accompanied by posterior and upward displacement of the anterior leaflet portion of the annulus from aortic root expansion during isovolumic systole. The effects of these changes on the anterior leaflet portion of the mitral annulus are to cause steepening and narrowing of the subaortic portion of the mitral annulus, which is attached to the aortic-mitral continuity. This is the "steepening of the saddle." These changes displace the anterior leaflet upward and posteriorly, widening the left ventricular outflow tract in early systole. The anisotropic systolic stretching of the anterior leaflet toward the posterior leaflet and the radial flattening of the anterior leaflet all further contribute to a progressive increase in the zone of apposition of the mitral leaflets. ${ }^{8,9,20-22}$

The effects of these changes are to produce progressively greater leaflet apposition as systole progresses (Figure 4). The beneficial effects of these phenomena have been studied in mathematical simulations and animal models. When functioning normally, these changes produce marked reduction in leaflet and chordal stresses. ${ }^{18,19}$ Kunzelman, Reimink, and Cochran ${ }^{18}$ simulated the effects of annular dilatation on leaflet apposition and stress distribution. They found that annular dilatation produced delayed and incomplete leaflet apposition, which was associated with markedly ele- vated stress on the chordae tendineae at all sites. Salgo, ${ }^{20}$ Sacks, ${ }^{21}$ Sakamoto, ${ }^{22}$ and their associates studied the influence of flat versus curved geometry of the annulus on stress pattern in the mitral valve. They showed marked reduction in leaflet stress in the "shaped annulus" model versus the "flat annulus."

The importance of using a repair technique that minimizes stress on the mitral valve components is illustrated by failure modes of other repair techniques that routinely include instances of annuloplasty ring dehiscence, leaflet suture line disruptions, failure of transposed chordae, and " progression of disease" in leaflets or chordae. ${ }^{4-8,23,24}$

The structural changes in myxomatous mitral valves were studied by Barber and associates. ${ }^{25}$ They found serious reduction of chordal strength, especially of the marginal chordae. Reduction of strength up to $90 \%$ has been reported. Thus, seriously weakened chordae must be presumed to be present at the time of the initial operation, and steps should be taken then to minimize the stress loadings these components will experience in the future.

As the foregoing studies indicate, the main mechanism that reduces stress in a normally functioning mitral valve is the presence of an adequate zone of leaflet apposition. The creation of a stable zone of apposition requires first that the edges of the leaflets be accurately aligned. This can be achieved easily with PTFE artificial chordae. However, the magnitude of the zone of apposition (defined as millimeters of leaflet contact measured from the free edge) is determined primarily by the geometry of the mitral annulus and its alterations during late diastole and throughout systole as the left ventricle is distended passively and then empties actively.

These changes cannot be assessed accurately in an arrested, collapsed heart because the changes during the cardiac cycle of the length and width of the left ventricle, the position of the annulus and the papillary muscles, and the 
early systolic aortic root enlargement all affect the position and shape of the mitral annulus and leaflets. The annulus itself varies in its 3-dimensional shape during the cardiac cycle.

For many years, as originally described by Carpentier, ${ }^{3}$ we had inflated the left ventricle with saline during mitral valve repair. Initially, we did this only to test the competence of the repair by simulating peak systolic conditions. However, we soon observed its utility in assessing end-diastolic and isovolumic systolic phenomena, as well as simulating some of the cyclical dynamic behavior of the mitral annulus in terms of the variations in its 3-dimensional shape, 3-dimensional area, and its descent into the left ventricle. We could also observe the corresponding changes in the leaflets in regard to edge alignment, leaflet curvature, and the systolic descent of the leaflets into the left ventricle. We hypothesized that since patients with no MR but severe myxomatous enlargement of the leaflets and normal annular dimensions do not have SAM, accurate adjustment of these relationships should avoid SAM without the need for leaflet resection under any circumstances. ${ }^{13,15,17}$ In the course of these observations of the mitral valve during inflation of the left ventricle, we found that a series of distinct and reproducible phenomena always occurred. We have now observed these in over 1000 patients.

These changes are illustrated in Figure 5, $A$ to $H$. The initial appearance of the severely prolapsing valve in the resting state is illustrated in Figure 5, A. After the edges of the mitral leaflets have been accurately aligned with PTFE chordae, the left ventricle is inflated at $4 \mathrm{~L} / \mathrm{min}$ with a laparoscopic suction irrigation device. If the mitral annulus is left in its resting dilated state $(55 \mathrm{~mm}$ in this case), the leaflets are displaced progressively into the left atrium as the left ventricle fills, producing a large tenting area above the plane of the mitral annulus The leaflets are very curved at this point and still look strikingly redundant. The valve is competent but the zone of apposition has minimal area. The mitral annulus dilates and moves upward toward the left atrium (Figure 5, $B$ ). Continued inflation eventually leads to marked leaflet bulging in the left atrium and ultimately central MR along the line of leaflet apposition, which is now very minimal in size (1-2 mm) (Figure 5, C).

If the posterior mitral annulus now is displaced forward toward the aortic root to simulate the atrially mediated reduction of $25 \%$ of the annular area at the end of diastole, inflation initially produces some bulging of the leaflets toward the atrium above the plane of the annulus, as previously observed without annular traction. However, with the annulus reduced in size, progressive inflation of the left ventricle now produces a distinctly different sequence of changes in the position of the leaflets. As shown by the change in position of the dots on the leaflets (Figure 5,D), a substantial amount of the leaflets has now been transferred from a plane parallel to the annulus to a plane parallel to the left ventric- ular long axis This represents development of an increase in the area of the zone of apposition between the 2 leaflets. This greatly reduces the projected areas of the leaflets acting as "sails" during systole. This corresponds to the normal position of the leaflets during left atrial contraction. As ventricular inflation is continued until systolic pressure is achieved in the left ventricle and aortic root (Figure 5, E and $G$ ), the leaflets now flatten (become less curved radially) and descend below the plane of the mitral annulus. The mitral annulus also noticeably descends away from the surgeon toward the apex of the left ventricle. The position of the dots shows that the leaflet area of apposition has increased, further reducing the projected area across the mitral orifice. Continued inflation now produces no MR. The leaflets are firmly locked together and difficult to separate (Figure 5,F).

Sizing for the annuloplasty ring is performed at this point, which corresponds to end-diastolic or early systolic dimensions. The latter "systolic" phase of this simulation is seen even better after the flexible ring annuloplasty has been performed, where the appearances of the early (Figure 5, $G$ ) and late phases (Figure 5, $H$ ) of inflation can be compared. The leaflets are more flattened axially and they assume a more vertical position radially. Tenting area is now absent. The anterior annulus can be seen to have steepened. These are the systolic changes in leaflet and annular shape documented by Sacks, ${ }^{21}$ Sakamoto, ${ }^{22}$ and their associates. Using 3-dimensional echocardiographic techniques with annular tracking software, we conducted a prospective study comparing patients operated on with these dynamic techniques with those operated on with the modified Carpentier technique at another institution during this same period. Detailed studies of annular and leaflet motion indicated that the techniques reported here provided superior maintenance of annular dynamic motion throughout the cardiac cycle, as well as preserved leaflet motion. ${ }^{6}$ The area of the annuloplasty ring was at a maximum during mid-diastole and decreased during systole to a minimum at mid-systole. ${ }^{26}$ The areas achieved were in the range of normal subjects (Table 2).

All the experimental and mathematical modeling suggests that the technique we have used should produce markedly reduced stress levels on the native chordal leaflets and the mitral annulus. The complete absence of annuloplasty ring dehiscence in our experience is a finding unique to this series. Suri and colleagues ${ }^{24}$ reported ring dehiscence or artificial chordal disruption as a cause for $29 \%$ of their 145 reoperations and progression of disease was recorded in $55 \%$ of patients. In our own experience, of the patients with myxomatous disease and recurrent MR, only a small minority have had evidence of "new" disease.

Our approach has been developed on the basis of the best available knowledge of the structure and function of the mitral valve in health and disease. In particular, we have attempted to incorporate all factors known to produce a low-stress repair. Better understanding of the role of the 

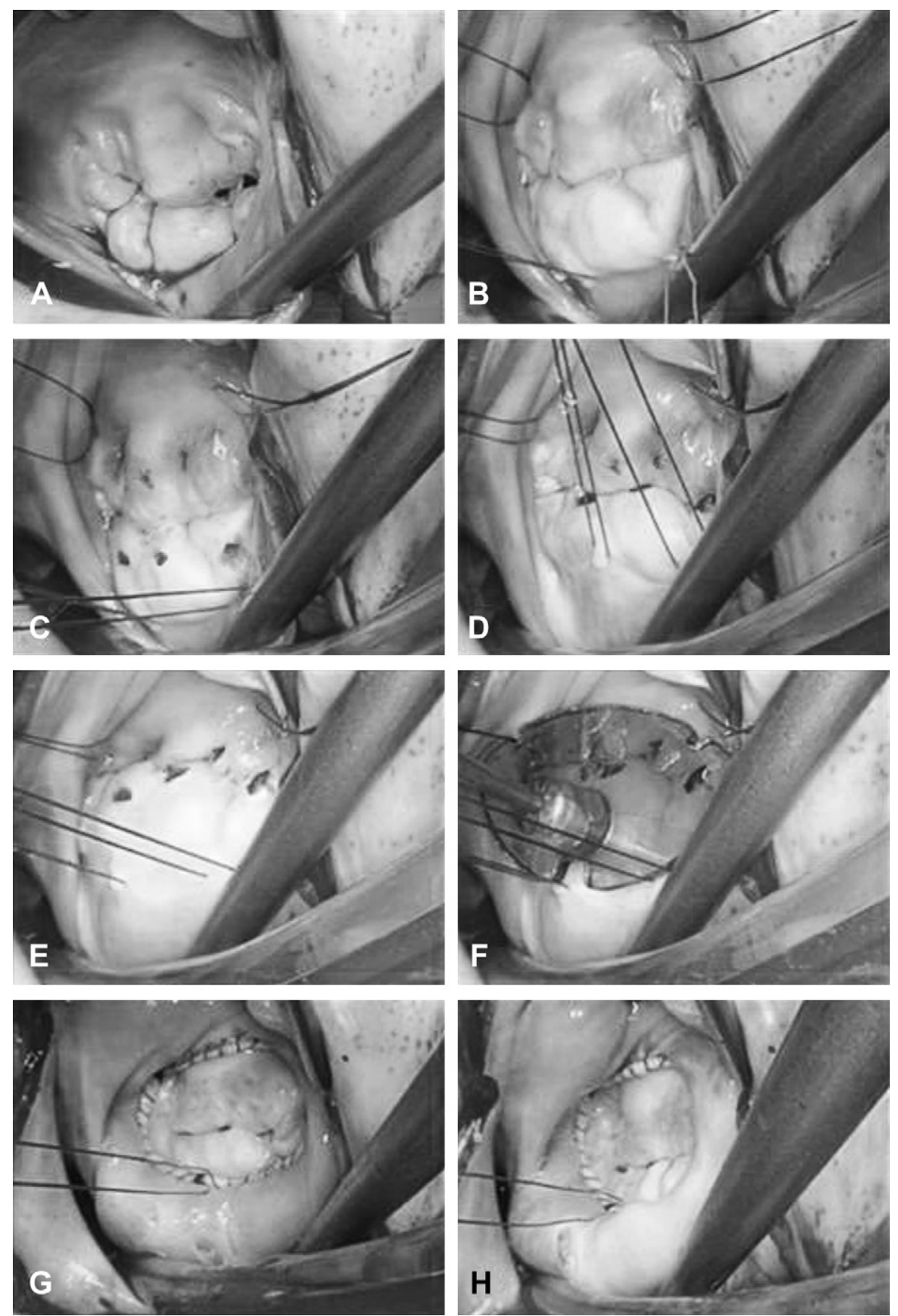

FIGURE 5. A, Initial appearance of a severely myxomatous valve with bileaflet involvement and annular dilatation. B, Inflation of the left ventricle without annular support causes increasing leaflet prolapse and annular dilatation. C, Leaflet edges are aligned. Dots are placed to mark upper edge of zone of apposition. D, When the posterior annulus is advanced with the left ventricle inflated, the leaflets develop the zone of apposition defined by the dots and descend into the left ventricle. E, The posterior annulus is pulled forward until the dots on each leaflet are aligned. F, This position is maintained and the sizer that matches this dimension is chosen. G, After the ring is attached, inflation causes the leaflets to flatten and enter the ventricle. $\mathrm{H}$, With inflation continued to 80 to $100 \mathrm{~mm}$ and the aortic root also inflated, the leaflets slope downward and the saddle shape of the anterior leaflet and annulus is developed.

mitral annulus in normal mitral valve function is central to these efforts. We believe the results reported here suggest that more attention should be directed to ensuring optimal, low-stress function of the mitral valve after repair, not simply the absence of MR.
We believe that the results achieved so far support the use of mechanically assisted pressurized inflation of the left ventricle, specifically to simulate end-diastolic and early systolic changes in mitral annular, chordal, and leaflet geometry as well as aortic root expansion as a setting for 
adjustment of PTFE chordal length and choice of appropriate annuloplasty ring. We have reported on our intraoperative observations of the reproducible changes seen intraoperatively with inflation of the left ventricle and manipulation of the mitral annulus and provided correlation for their explanation from the literature on mitral valve function.

The experience reported here has confirmed our hypothesis that leaflet resection is not required to avoid SAM, regardless of leaflet morphology. SAM is prevented, despite large leaflets, by accurate dynamic leaflet positioning and annular sizing during ventricular inflation. The annular size has been restored to within the range of normal dimensions after correction by this method (Table 2).

We have demonstrated the utility of the concept of first performing leaflet edge alignment by chordal length and the separate adjustment of the area of the leaflet zone of apposition, which is determined largely by annular size.

Routine $100 \%$ reparability for myxomatous and degenerative mitral valve disease has been achieved using these techniques. The success rates for anterior and bileaflet repairs have been similar to those of posterior leaflet repairs.

The preliminary results of ongoing studies based on 3-dimensional echocardiography and magnetic resonance imaging evaluation of these patients in our institution have provided support for these concepts. ${ }^{26}$ Further refinement or modification of these techniques may well be required as further data are acquired on postoperative mitral valve morphology and function from these studies.

\section{Limitation}

The principal limitation of this study is the fact that due to a wide geographic referral area, it has not been possible to perform all follow-up echocardiograms in our own echocardiography laboratory. Echocardiographic follow-up was also obtained in only $71 \%(469 / 662)$ of patients.

\section{References}

1. McGoon DC. Repair of mitral insufficiency due to ruptured chordae tendineae. J Thorac Cardiovasc Surg. 1960;39:357-9.

2. Carpentier A. Pathology of the mitral valve. In: Kalmanson D, ed. The mitral valve: pluridisciplinary approach. Acton (MA): Publishing Sciences Group Inc; 1976. p. 65-77.

3. Carpentier A. Plastic and reconstructive mitral valve surgery. In: Kalmanson D, ed. The mitral valve: pluridisciplinary approach. Acton (MA): Publishing Sciences Group Inc; 1976. p. 527-40.

4. David TE, Ivanov J, Armstrong S, Christie D, Rakowski H. A comparison of outcomes of mitral valve repair for degenerative disease with posterior, anterior, and bileaflet prolapse. J Thorac Cardiovasc Surg. 2005;130:1242-9.

5. Flameng W, Meuris B, Herijgers P, Herregods M. Durability of mitral valve repair in Barlow disease versus fibroelastic deficiency. J Thorac Cardiovasc Surg. 2008; 135:274-82.
6. Chung CH, Kim JB, Choo SJ, Kim KS, Song H, Son MG, et al. Long-term outcomes after mitral ring annuloplasty for degenerative mitral regurgitation: Duran ring versus Carpentier-Edwards ring. J Heart Valve Dis. 2007;16:536-45.

7. Chang BC, Youn YN, Ha JW, Lim SH, Hong YS, Chung N. Long-term clinical results of mitral valvuloplasty using flexible and rigid rings: a prospective and randomized study. J Thorac Cardiovasc Surg. 2007;133:995-1003.

8. Tsakiris AG, Bernuth G, Rastelli GC, Bourgeois MJ, Titus JL, Wood EH. Size and motion of the mitral valve annulus in anesthetized intact dogs. J Appl Physiol. 1971;30:611-8.

9. Fann JI, Ingels NB, Miller DC. Pathophysiology of mitral valve disease. In: Cohn LH, Edmunds LH Jr., eds. Cardiac surgery in the adult. New York: McGraw-Hill; 2003. p. 901-31.

10. Lawrie GM, Earle EA, Earle NR. Feasibility and intermediate term outcome of repair of prolapsing anterior mitral leaflets with artificial chordal replacement in 152 patients. Ann Thorac Surg. 2006;81:849-56.

11. Shuhaiber J, Anderson RJ. Meta-analysis of clinical outcomes following surgical mitral valve repair or replacement. Eur J Cardiothorac Surg. 2007;31:267-75.

12. Wooley CF, Baker PB, Kolibash AJ, Kilman JW, Sparks EA, Boudoulas H. The floppy myxomatous mitral valve, mitral valve prolapse, and mitral regurgitation. Progr Cardiovasc Dis. 1991;32:397-433.

13. Pini R, Devereaux RB, Greppi B, Roman MJ, Hochreiter C, Kramer-Fox R, et al. Comparison of mitral valve dimensions and motion in mitral valve prolapse with severe mitral regurgitation to uncomplicated mitral valve prolapse and to mitral regurgitation without mitral valve prolapse. Am J Cardiol. 1988; 62:257-63.

14. Avierinos JR, Gersh BJ, Melton J III, Bailey KR, Shub C, Nishimura RA, et al. Natural history of asymptomatic mitral valve prolapse in the community. Circulation. 2002;106:1355-61.

15. Freed LA, Benjamin EJ, Levy D, Larson MG, Evans JC, Fuller DL, et al. Mitral valve prolapse in the general population. J Am Coll Cardiol. 2002;40: 1298-304.

16. Freed LA, Levy D, Levine RA, Larson MG, Evans JC, Fuller DL, et al. Prevalence and clinical outcome of mitral valve prolapse. N Engl J Med. 1999;341:1-7.

17. Ormiston JA, Pravin CB, Shah PM, Tei C, Wong M. Size and motion of the mitral valve annulus in man. II. Abnormalities in mitral valve prolapse. Circulation. 1982;65:713-9.

18. Kunzelman KS, Reimink MS, Cochran RP. Annular dilatation increases stress in the mitral valve and delays coaptation; a finite element computer model. Cardiovasc Surg. 1997;5:427-34.

19. Nazari S, Carli F, Salvi S, Banfi C, Aluffi A, Mourad Z, et al. Patterns of systolic stress distribution on mitral valve anterior leaflet chordal apparatus. Cardiovasc Surg. 2000;41:193-202.

20. Salgo IS, Gorman JH, Gorman RC, Jackson BM, Bowen FW, Plappert T, et al. Effect of annular shape on leaflet curvature in reducing mitral leaflet stress. Circulation. 2002;106:711-7.

21. Sacks MS, Enomoto Y, Graybill JR, Merryman WD, Zeeshan A, Yoganathan AP, et al. JH. In-vivo dynamic deformation of the mitral valve anterior leaflet. Ann Thorac Surg. 2006;82:1369-77.

22. Sakamoto H, Parish LM, Hamamoto H, Enomoto Y, Zeeshan A, Plappert T, et al. Effects of hemodynamic alterations on anterior mitral leaflet curvature during systole. J Thorac Cardiovasc Surg. 2006;132:1414-9.

23. Gillinov AM, Cosgrove DM, Blackstone EH, Diaz R, Arnold JH, Lytle BW, et al. Durability of mitral repair for degenerative disease. J Thorac Cardiovasc Surg. 1998;116:734-43.

24. Suri RM, Schaff HV, Dearani JA, Sundt TM, Daley RC, Mullany CJ, et al. Recurrent mitral regurgitation after repair: should the mitral valve be re-repaired? J Thorac Cardiovasc Surg. 2006;132:1390-7.

25. Barber JE, Kasper RK, Ratliff NB, Cosgrove DM, Griffin BP, Vesely I. Mechanical properties of myxomatous mitral valves. J Thorac Cardiovasc Surg. 2001; 122:955-62.

26. Zekry SB, Lang RM, Sugeng L, McCulloch ML, Weinert L, Ramen J, et al. Annular structure and function in organic mitral regurgitation: effect of valve repair and comparison of the modified Carpentier method with a new "American Correction."' Circulation. 2009;120:S769. 